\title{
ON CURVED SHOCK WAVES IN THREE-DIMENSIONAL GAS FLOWS*
}

\author{
BY \\ R. P. KANWAL** \\ Indiana University
}

Introduction. In this paper we discuss the problem of shock waves in threedimensional steady rotational flows of an ideal gas with viscosity and heat conduction neglected and subject to no extraneous force. If a shock wave arises, e.g., as the result of the presence of an obstacle in the field of the flow, it will be assumed that this divides the flow under consideration into a region 1 and a region 2 . We suppose region 1 to be traversed by the gas before contact with the shock surface and region 2 to be that into which gas enters after passing through the shock surface. It is assumed that this surface is given by a continuous and differentiable function of coordinates and that it has a continuous and differentiable unit normal which we suppose directed from region 1 to region 2 . Furthermore, we assume the required differentiability conditions to be satisfied by the velocity components $u_{i}$, the density $\rho$, pressure $p$ and entropy $S$ in each region 1 and 2 so that it is possible to express the surface covariant derivatives of these quantities along either side of the shock surface.

If the flow in front of the shock is known then the flow behind the shock is determined by the well-known Rankine-Hugoniot relations. The main object of this paper is to obtain formulas for the determination of the gradients of velocity components, pressure, density and entropy behind the shock surface when the flow in front is known. These formulas have been obtained for plane flows by Thomas [1]. In his analysis Thomas assumed that $\gamma$, the ratio of specific heats, is constant throughout the flow. Truesdell [2] has extended these results to fluids obeying an arbitrary equation of state. The same can be done for the three-dimensional gas flows also, although we have carried out the calculations for the case when $\gamma$ is constant. Truesdell has further put Thomas's results in more compact form by introducing certain dimensionless variables which have been found useful in the following discussion also.

The required derivatives are obtained by differentiating the Rankine-Hugoniot relations along the shock surface. Gauss-Weingarten formulas and various other results of the geometry of surfaces concerning the principal normal curvatures are found useful in the analysis. The complete analysis has been given only for the case of uniform flow upstream of the shock. As anticipated, the flow behind the shock wave is found in general to be rotational. The explicit determination of the vorticity components is carried out. This leads to the formulation of a theorem regarding the characterization of surfaces behind which the flow remains irrotational. It is found that the plane, the right circular cone, the cylinder and the developable helicoid are the only such surfaces. Furthermore, it is found that the component of vorticity along the normal to the shock surface vanishes at every point of the surface - a property shared by the contact discontinuities which

\footnotetext{
*Received August 26, 1957. This work was done under N.S.F. Contract No. G2256, with Indiana University. It forms part of the author's doctoral thesis in applied mathematics, Indiana University, 1957. Part of the results were presented at the meeting of American Mathematical Society at Chicago in April 1957.

**Present address: Mathematics Research Center, U. S. Army, University of Wisconsin.
} 
are surfaces across which the normal component, of the velocity is continuous while the tangential components are discontinuous.

Formal methods of tensor analysis have been used throughout the following discussion.

1. Equations of motion. We consider the differential relations governing the steady flow of a perfect gas devoid of viscosity and heat conduction and subject to no extraneous force, namely $[3,4]$,

$$
\begin{array}{ll}
p_{, i}+\rho u_{j} u_{i, j}=0, & \text { (equations of motion), } \\
\rho_{, i} u_{i}+\rho u_{i, j}=0, & \text { (equation of continuity), }
\end{array}
$$

in which $p, \rho$ and $u_{i}$ denote the pressure, density and velocity components respectively. We assume the motion referred to a system of rectangular coordinates $x^{i}$; then the comma in the above equations and in the following, represents partial differentiation. It is to be understood in the above and in the following discussion, unless the contrary is stated, that an index which occurs twice in a term is to be summed over the admissable values of the index. Since there is no distinction between covariant and contravariant indices within a rectangular system, we may write any index as a subscript or a superscript without modifying the value of the term in which the index occurs.

In addition there is the equation of state, viz. [3, 4],

$$
p=\exp \left(S / J c_{v}\right) \rho^{\gamma},
$$

where $S$ is the entropy, $J$ the mechanical equivalent of heat and $\gamma$ is the ratio of two specific heats $c_{p}$ and $c_{v}$ assumed constant. The entropy remains constant on a streamline but varies from streamline to streamline i.e., $S_{i} u_{i}=0$. By differentiating (3) and using the Eqs. (1) and (2) we obtain

$$
u_{i, j} u_{i} u_{i}-c^{2} u_{k, k}=0,
$$

where $c^{2}=\gamma p / \rho$, is the adiabatic speed of sound.

2. The Rankine-Hugoniot relations. Denote by $u_{1 i}, \rho_{1}$ and $p_{1}$ the velocity components, density and pressure on the side of the wave bordering region 1 and correspondingly by $u_{2 i}, \rho_{2}$ and $p_{2}$ the values of these quantities on the side of the wave bordering region 2. Put

$$
u_{1 n}=u_{1 i} \xi^{i}, \quad u_{2 n}=u_{2 i} \xi^{i},
$$

so that $u_{1 n}$ and $u_{2 n}$ are the normal components of the velocity on sides 1 and 2 of the wave surface, respectively. Then the Rankine-Hugoniot relations for the stationary wave under consideration can be written as

$$
\begin{aligned}
{\left[u_{i}\right] } & =\frac{2\left(\rho_{1} u_{1 n}^{2}-\gamma p_{1}\right) \xi_{i}}{-(\gamma+1) \rho_{1} u_{1 n}}, \\
{[p] } & =\frac{2\left(\rho_{1} u_{1 n}^{2}-\gamma p_{1}\right)}{(\gamma+1)}, \\
{[\rho] } & =\frac{2 \rho_{1}\left(\rho_{1} u_{1 n}^{2}-\gamma p_{1}\right)}{2 \gamma p_{1}+(\gamma-1) \rho_{1} u_{1 n}^{2}} .
\end{aligned}
$$

Here the bracket [ ] denotes the difference of the values on the two sides of the shock surface of the quantity enclosed, e.g., $\left[u_{i}\right]=u_{2 i}-u_{1 i}$. 
3. Coordinate system on the shock surface. In the following analysis it has been found convenient and advantageous to have the lines of curvature as the Gaussian coordinate curves on the shock surface. Denote these coordinates by $y^{1}, y^{2}$. Then the lines of curvature are $y^{1}=$ constant and $y^{2}=$ constant and the parametric equations of the surface are given by

$$
x^{i}=x^{i}\left(y^{1}, y^{2}\right), \quad i=1,2,3 .
$$

As is customary we shall use Latin letters for the indices referring to the space variables and Greek letters for the indices referring to the surface variables. Thus the Latin indices will assume values 1,2 and 3, and Greek indices the values 1 and 2 .

The unit surface tangent vectors to the coordinate curves are [5a] $\delta_{1}^{\alpha} g_{11}$ and $\delta_{2}^{\alpha} g_{22}$ respectively where

$$
g_{\alpha \beta}=1 /\left(a_{\alpha \beta}\right)^{1 / 2},
$$

and $a_{\alpha \beta}$ are the components of the first fundamental form of the surface

$$
a_{\alpha \beta}=\frac{\partial x^{i} \partial x^{i}}{\partial y^{\alpha} \partial y^{\beta}}
$$

while $\delta$ is the Kronecker delta. The corresponding space components of the unit tangent vectors are $x_{1}^{i} g_{11}$ and $x_{2}^{i} g_{22}$ respectively, where we have put

$$
x_{\alpha}^{i}=\frac{\partial x^{i}}{\partial y^{\alpha}} .
$$

Then $x_{\alpha}^{i}$ are the components of a contravariant space vector and a covariant surface vector. We shall call these quantities the components of projection tensor [6]. We have already assumed that the normal vector to the shock surface is directed from region 1 to region 2. We further choose the orientation of the surface normal and Gaussian coordinates in such a way that $x_{1}^{i}, x_{2}^{i}$ and $\xi^{i}$ have the same orientation as the $x^{1}-, x^{2}-$ and $x^{3}$-axis.

Now consider the velocity field $u_{i}$ and let $\zeta_{i}$ denote the part of the velocity field which is normal to the surface and let $v_{i}$ denote that part of $u_{i}$ which lies in the tangent plane of the surface. Evidently we have

$$
u_{i}=v_{i}+\zeta_{i} .
$$

By multiplying both sides of this relation by $x_{\alpha}^{i}$ we obtain [6]

$$
x_{\alpha}^{i} u_{i}=v_{\alpha},
$$

where we have put $x_{\alpha}^{i} v_{i}=v_{\alpha}$. We say that the tensor $x_{\alpha}^{i}$ projects $u_{i}$ onto the tangent plane to the surface, $v_{\alpha} g_{\alpha \alpha}$ ( $\alpha$ not summed) being the tangential component of the velocity along the $y^{\alpha}$ curve.

Following Truesdell [2] we introduce the dimensionless variables:

$$
\begin{array}{ll}
\delta=\frac{2\left(\rho_{1} u_{1 n}^{2}-\gamma p_{1}\right)}{2 \gamma p_{1}+(\gamma-1) \rho_{1} u_{1 n}^{2}}, & \tau_{\alpha}=\frac{v_{\alpha} g_{\alpha \alpha}}{u_{1 n}}, \\
\chi_{\alpha}=\frac{v_{\alpha} g_{\alpha \alpha}}{u_{2 n}}, \quad M_{1 n}=\frac{u_{1 n}}{c_{1}}, & M_{2 n}=\frac{u_{2 n}}{c_{2}},
\end{array}
$$


where $\alpha$ is not summed, $\delta$ is the shock strength and $\tau_{\alpha}$ are the components of the obliquity of the shock. With these abbreviations the shock relations can be written as

$$
\begin{aligned}
{\left[u_{i}\right] } & =\frac{-\delta u_{1 n} \xi_{i}}{1+\delta}, \\
{[p] } & =\frac{\delta}{1+\delta} \rho_{1} u_{1 n}^{2}, \\
{[\rho] } & =\delta \rho_{1} .
\end{aligned}
$$

Multiplying both sides of (13) by $\xi_{i}$ and $x_{\alpha}^{i}$ we easily deduce

$$
u_{2 n}=\frac{u_{1 n}}{1+\delta}=\frac{\rho_{1} u_{1 n}}{\rho_{2}}
$$

and

$$
v_{2 \alpha}=v_{1 \alpha} .
$$

4. Differentiation of the shock relations. Differentiating the relations (5) to (7) with respect to $y^{1}$ and $y^{2}$ we get relations along the shock surface of the form

$$
\begin{aligned}
u_{i, j} x_{\alpha}^{j} & =u_{1 i, j} x_{\alpha}^{j}+A_{i \alpha}=A_{i \alpha}^{*}, \\
p_{, j} x_{\alpha}^{j} & =p_{1, i} x_{\alpha}^{j}+B_{\alpha}=B_{\alpha}^{*}, \\
\rho_{, j} x_{\alpha}^{j} & =\rho_{1, j} x_{\alpha}^{j}+C_{\alpha}=C_{\alpha}^{*},
\end{aligned}
$$

where for simplicity we have omitted the subscript 2 on the quantities appearing in the left members of these equations. The explicit formulas for the quantities $A_{i \alpha}, B_{\alpha}$ and $C_{\alpha}$ are readily obtained by differentiating the right members of the equations (5) to (7), respectively, with the help of the Gauss-Weingarten formulas of differential geometry (see Sec. 6). We see that these quantities are expressible in terms of the known parameters of the flow in the region 1 and their partial derivatives along the shock surface, the projection tensor $x_{\alpha}^{i}$, the surface normal $\xi$ and the two principal curvatures. Following Thomas [1], any quantity which can be so expressed will be said, in the following, to have an allowable determination.

5. Derivation of the partial derivatives behind the shock surface. Consider the Eqs. (1), (2), (4), (18), (19), and (20). From this set of six relations we can determine the partial derivatives of the velocity components $u_{i}$, the density $\rho$ and the pressure $p$ immediately behind the shock surface. For this purpose we first eliminate the derivatives $p_{. i}$ to obtain the following equations, which we represent as two sets of equations for convenience of reference, namely

$$
\begin{gathered}
\rho_{, j} x_{\alpha}^{j}=C_{\alpha}^{*}, \\
\rho_{, j} u_{i}+\rho u_{k, k}=0,
\end{gathered}
$$

and

$$
\begin{aligned}
u_{i, j} x_{\alpha}^{j} & =A_{i \alpha}^{*}, \\
\rho u_{i, j} x_{\alpha}^{i} u_{i} & =-B_{\alpha}^{*}, \\
\rho u_{i, j} u_{i} u_{i}-\gamma p u_{k, k} & =0 .
\end{aligned}
$$


Now from (23) to (25) we can obtain $u_{i, j}$ and then from (21) and (22) $\rho_{, i}$ can be found; after this the Eqs. (1) yield the values of the derivatives $p_{. i}$. To effect the above determination we define a matrix $\left\|C_{i i}\right\|$ such that

$$
C_{i \alpha}=\lambda_{i \alpha} ; \quad C_{i 3}=u_{i} / u_{n}
$$

where $\lambda_{i \alpha}$ denote the space components of the unit tangent vector to the curve $y^{\alpha}$ and are given, as remarked above, in terms of the projection tensor by

$$
\lambda_{i \alpha}=\lambda_{\alpha}^{i}=x_{\alpha}^{i} g_{\alpha \alpha}, \quad \text { no summation on } \alpha \text {. }
$$

The determinant of the matrix $\left\|C_{i}\right\|$ is easily seen to be unity. Hence we can define the quantities $D_{\boldsymbol{i k}}$ by

$$
D_{i k} C_{k i}=\delta_{i i} ; \quad D_{k i} C_{i k}=\delta_{i i} .
$$

The second relation (27) follows from the first relation and conversely. Since we shall have to use the explicit form of the matrix $\left\|D_{i j}\right\|$, it can be easily seen to be given by

$$
\left\|\begin{array}{lll}
D_{11} & D_{21} & D_{31} \\
D_{12} & D_{22} & D_{32} \\
D_{13} & D_{23} & D_{33}
\end{array}\right\|=\left\|\begin{array}{lll}
\frac{\left(\lambda_{2}^{2} u_{3}-\lambda_{2}^{3} u_{2}\right)}{u_{n}} & \frac{\left(\lambda_{1}^{3} u_{2}-\lambda_{1}^{2} u_{3}\right)}{u_{n}} & \xi_{1} \\
\frac{\left(\lambda_{2}^{3} u_{1}-\lambda_{2}^{1} u_{3}\right)}{u_{n}} & \frac{\left(\lambda_{1}^{1} u_{3}-\lambda_{1}^{3} u_{1}\right)}{u_{n}} & \xi_{2} \\
\frac{\left(\lambda_{2}^{1} u_{2}-\lambda_{2}^{2} u_{1}\right)}{u_{n}} & \frac{\left(\lambda_{1}^{2} u_{1}-\lambda_{1}^{1} u_{2}\right)}{u_{n}} & \xi_{3}
\end{array}\right\| .
$$

Now define the quantities $B_{i j}$ by

$$
B_{i j}=u_{l, m} C_{l i} C_{m i} .
$$

Then from (23), (24), (25) and (29) we have

$$
\begin{aligned}
B_{\alpha \beta}=x_{\alpha}^{i} A_{i \beta}^{*} g_{\alpha \alpha} g_{\beta \beta}, & B_{\alpha 3}=-\frac{B_{\alpha}^{*} g_{\alpha \alpha}}{\rho \iota_{n}}, \\
B_{3 \alpha}=\frac{A_{\alpha \alpha}^{*} u_{i} g_{\alpha \alpha}}{u_{n}}, & B_{33}=\frac{u_{k, k}}{M_{n}^{2}},
\end{aligned}
$$

where $\alpha, \beta$ are not summed.

Furthermore, by multiplying both sides of (29) by $D_{i,} D_{i l}$ and using the relation (27) we readily obtain

$$
u_{l, m}=B_{i j} D_{i l} D_{j m} .
$$

Now the first eight elements of the matrix $\left\|B_{i i}\right\|$ have an allowable determination as can be readily seen. However, $B_{33}$ is given in terms of the $u_{k k}$; it therefore remains to determine the quantity $B_{33}$. But this can easily be done by contracting the indices $l$ and $m$ in (31), viz.,

$$
u_{k, k}=B_{i j} D_{i k} D_{i k},
$$

which, by the help of the matrix (28), gives the value

$$
u_{k, k}=B_{11}\left(1+\chi_{1}^{2}\right)+B_{22}\left(1+\chi_{2}^{2}\right)+2 B_{(12)} \chi_{1} \chi_{2}-2 B_{(13)} \chi_{1}-2 B_{(23)} \chi_{2}+B_{33},
$$


where $\chi$ 's are defined by (12) and for brevity we have introduced

$$
B_{(i i)}=\frac{1}{2}\left(B_{i j}+B_{i i}\right) \text {. }
$$

Thus we arrive at the value of $B_{33}$

$$
B_{33}=\frac{B_{11}\left(1+\chi_{1}^{2}\right)+B_{22}\left(1+\chi_{2}^{2}\right)+2 B_{(12)} \chi_{1} \chi_{2}-2 B_{(13)} \chi_{1}-2 B_{(23)} \chi_{2}}{M_{n}^{2}-1},
$$

which furnishes an allowable determination of $B_{33}$. Hence the relations (31), in which $B_{33}$ is given by (32), provide an allowable determination of the gradients of velocity components behind the shock wave.

To effect the determination of the gradient of density behind the shock surface we observe that the set of equations (22) and (23) can be written in the form

$$
\rho_{, i} C_{i i}=d_{i} \text {, }
$$

where

$$
\begin{aligned}
& d_{1}=g_{11} C_{1}^{*}, \quad d_{2}=g_{22} C_{2}^{*}, \\
& d_{3}=-\rho u_{k, k} / u_{n}=-\rho B_{33} M_{n}^{2} / u_{n} .
\end{aligned}
$$

Hence we have

$$
\rho_{, j}=d_{i} D_{i j}
$$

as the allowable determination of the quantities $\rho_{. i}$. Finally from (1) the derivatives $p_{. i}$ behind the shock surface can be determined by the equations

$$
p_{, i}=-\rho u_{i} u_{i, j}=-\rho u_{n} B_{i 3} D_{i i} .
$$

6. Calculation of the invariants $d_{i}$ and $B_{i j}$. It is possible to give an explicit formulation of the invariants $d_{i}$ and $B_{i i}$ under the assumption that the flow in front of the shock is uniform, i.e., the velocity, pressure, and density are constant in region 1 . To effect this formulation we need some results of the differential geometry which are briefly stated below [5a].

We have already noted the expressions for the first fundamental form of the surface, the projection tensor and the space components of the unit tangent vectors to the coordinate curves. They are

$$
a_{\alpha \beta}=x_{\alpha}^{i} x_{\beta}^{i}, \quad x_{\alpha}^{i}=\frac{\partial x^{i}}{\partial y^{\alpha}}, \quad \lambda_{\alpha}^{i}=g_{\alpha \alpha} x_{\alpha}^{i}, \quad \alpha \text { not summed },
$$

respectively. In addition we need the expression for the normal curvatures in the directions of the coordinate curves (which are the lines of curvatures and hence these curvatures are the principal normal curvatures). They are

$$
k_{1}=b_{11} / a_{11} ; \quad k_{2}=b_{22} / a_{22},
$$

where $b_{\alpha \beta}$ are the components of the second fundamental form of the surface, i.e.;

$$
b_{\alpha \beta}=\frac{1}{2} \epsilon^{\gamma \delta} e_{i j k} x_{\alpha ; \beta}^{i} x_{\gamma}^{j} x_{\delta}^{k} .
$$

In the above relation $\epsilon^{\gamma \delta}$ are the components of the surface permutation tensor with the properties 


$$
\epsilon^{11}=\epsilon^{22}=0 ; \quad \epsilon^{12}=1 /(a)^{1 / 2} ; \quad \epsilon^{21}=-1 /(a)^{1 / 2},
$$

where $a$ is the det $\left\|a_{\alpha \beta}\right\|$, while $e_{i j k}$ are the components of the permutation tensor of the space with the properties

$$
\begin{aligned}
& e_{i j k}=0 \text { if any two of the indices } i, j \text { and } k \text { are equal, } \\
& e_{123}=e_{231}=e_{312}=+1 \\
& e_{132}=e_{321}=e_{213}=-1,
\end{aligned}
$$

and $x_{\alpha ; \beta}^{i}$ denotes the surface covariant differentiation of $x_{\alpha}^{i}$. As we have taken the lines of curvature as the coordinate curves on the shock surface we have

$$
a_{12}=b_{12}=0 \text {. }
$$

Moreover if $a^{\alpha \beta}$ denote the components of the tensor conjugate to $a_{\alpha \beta}$, namely,

$$
a_{\alpha \beta} a^{\alpha \gamma}=\delta_{\beta}^{\gamma} \text {, }
$$

we obtain

$$
a^{11}=a_{22} / a, \quad a^{12}=a^{21}=0, \quad a^{22}=a_{11} / a .
$$

In this notation the space components of the unit normal vector to the surface are given by the relation

$$
\xi^{i}=\frac{1}{2} \epsilon^{\alpha \beta} e_{i j k} x_{\alpha}^{i} x_{\beta}^{k} .
$$

The well-known Weingarten formulas give

$$
\xi_{; \alpha}^{i}=-a^{\gamma \beta} b_{\alpha \gamma} x_{\beta}^{i},
$$

where ; denotes, as before, the surface covariant differentiation. Keeping these results and the assumption of uniform flow in front of the shock in mind, we get

$$
u_{1 n ; \alpha}=u_{1 i} \xi_{; \alpha}^{i}=-u_{1 i} a^{\gamma \beta} b_{\alpha \gamma} x_{\beta}^{i}=v_{\beta} a^{\gamma \beta} h_{\alpha \gamma},
$$

where we have used the relations (11) and (17). Expanding this result and making use of the expressions in (35) and (38) we obtain

$$
u_{1 n ; 1}=-v_{1} k_{1} ; \quad u_{1 n ; 2}=-v_{2} k_{2} .
$$

Similarly, the relation (40) yields

$$
\xi_{; 1}^{i}=-k_{1} x_{1}^{i} ; \quad \xi_{; 2}^{i}=-k_{2} x_{2}^{i} .
$$

Now for the uniform flow

$$
A_{i \alpha}^{*}=A_{i \alpha}, \quad B_{\alpha}^{*}=B_{\alpha}, \quad C_{\alpha}^{*}=C_{\alpha} .
$$

Hence we can readily find [7].

$$
\begin{aligned}
A_{\text {i } \alpha} & =\left\{\frac{2\left(\rho_{1} u_{1 n}^{2}-\gamma p_{1}\right) \xi_{i}}{-(\gamma+1) \rho_{1} u_{1 n}}\right\}_{i \alpha} \\
& =-\left\{\frac{B_{\alpha} \xi_{i}}{\rho_{1} u_{1 n}}+\frac{[p]}{\rho_{1} u_{1 n}^{2}}\left(-k_{\alpha} x_{\alpha}^{i} u_{1 n}+\xi^{i} v_{\alpha} k_{\alpha}\right)\right\},
\end{aligned}
$$


in which there is no summation on $\alpha$. From (14), (30), and (44) we obtain

$$
\begin{aligned}
& B_{11}=\frac{A_{i 1} x_{1}^{i}}{a_{11}}=\frac{[p]}{\rho_{1} u_{1 n}^{2}} k_{1} \cdot u_{1 n} \cdot \frac{x_{1}^{i} x_{1}^{i}}{a_{11}}=\delta u_{n} k_{1}, \\
& B_{12}=B_{21}=0, \\
& B_{22}=\delta u_{n} k_{2} .
\end{aligned}
$$

Also from (6) and (42) we get

$$
B_{\alpha}=\frac{-4 \rho_{1} u_{1 n} v_{\alpha} k_{\alpha}}{\gamma+1}, \quad \text { no summation on } \alpha,
$$

yielding

$$
B_{13}=-\frac{B_{1} g_{11}}{\rho u_{n}}=\frac{4 g_{11} v_{1} k_{1}}{\gamma+1},
$$

and

$$
B_{23}=-\frac{B_{2} g_{22}}{\rho u_{n}}=\frac{4 g_{22} v_{2} k_{2}}{\gamma+1},
$$

where the relation (16) viz., $\rho_{1} u_{1 n}=\rho_{2} u_{2 n}$ has been used.

Furthermore, from (44) we obtain

$$
A_{i \alpha} \frac{u_{i}}{u_{n}}=-\left\{B_{\alpha} \cdot \frac{1}{\rho_{1} u_{1 n}}+\frac{\delta}{1+\delta}\left(-v_{\alpha} k_{\alpha} \cdot \frac{u_{1 n}}{u_{n}}+v_{\alpha} k_{\alpha}\right)\right\},
$$

in which there is no summation on $\alpha$. From this we get

$$
B_{31}=\frac{A_{i 1} u_{i} g_{11}}{u_{n}}=v_{1} g_{11} k_{1}\left(\frac{4}{\gamma+1}+\frac{\delta^{2}}{1+\delta}\right),
$$

and

$$
B_{32}=\frac{A_{i 2} u_{i} g_{22}}{u_{n}}=v_{2} g_{22} k_{2}\left(\frac{4}{\gamma+1}+\frac{\delta^{2}}{1+\delta}\right) .
$$

Lastly from the formula (32) we get the value of $B_{33}$ as

$$
\begin{aligned}
B_{33}=\left\{\delta u_{n}\left(1+\chi_{1}^{2}\right)-\left(\frac{8}{\gamma+1}+\right.\right. & \left.\left.\frac{\delta^{2}}{1+\delta}\right) \chi_{1}^{2}\right\} k_{1} \\
& +\left\{\delta u_{n}\left(1+\chi_{2}^{2}\right)-\left(\frac{8}{\gamma+1}+\frac{\delta^{2}}{1+\delta_{2}^{2}}\right)\right\} k_{2} .
\end{aligned}
$$

The matrix $\left\|B_{i i}\right\|$ is thereby completely determined.

Correspondingly the $d_{i}$ are given by the expressions

$$
\begin{aligned}
& d_{1}=C_{1} g_{11}=\frac{2 g_{11} v_{1} k_{1}}{(\gamma+1) u_{n}}\left\{(\gamma-1) \rho-(\gamma+1) \rho_{1}\right\}, \\
& d_{2}=C_{2} g_{22}=\frac{2 g_{22} v_{2} k_{2}}{(\gamma+1) u_{n}}\left\{(\gamma-1) \rho-(\gamma+1) \rho_{1}\right\}, \\
& d_{3}=-\frac{\rho B_{33} M_{n}^{2}}{u_{n}} .
\end{aligned}
$$


From the relations (45) to (50) and the relations (51) the invariant character of $B_{i}$ and $d_{i}$ can be easily seen.

7. Variation of velocity, density and pressure along the shock surface. The rate of change with respect to the arc length along the lines of curvature of the velocity components $u_{i}$ is given by the following formula

$$
u_{i, j} x_{\alpha}^{i} g_{\alpha \alpha}=B_{l m} D_{l i} D_{m i} x_{\alpha}^{i} g_{\alpha \alpha}, \quad \alpha \text { not summed. }
$$

When expanded this becomes

$$
u_{i, i} \lambda_{1}^{i}=B_{l 1} D_{l i}, \quad u_{i, j} \lambda_{2}^{j}=B_{l 2} D_{l i} .
$$

Similarly the rate of change of density and pressure is given by

$$
\rho_{, i} \lambda_{\alpha}^{i}=d_{\alpha},
$$

and

$$
p_{, i} \lambda_{\alpha}^{i}=-\rho u_{n} B_{i 3} D_{i i} \lambda_{\alpha}^{i},
$$

which, when expanded, becomes

$$
p_{, i} \lambda_{1}^{i}=-\rho u_{n} B_{13}, \quad p_{,} \lambda_{2}^{i}=-\rho u_{n} B_{23} .
$$

From (52) we readily find

$$
\begin{aligned}
& q{ }_{, i} \lambda_{1}^{i}=u_{i} u_{i, i} \lambda_{1}^{i}=B_{31}, \\
& q{ }_{, i} \lambda_{2}^{i}=u_{i} u_{i, i} \lambda_{2}^{i}=B_{32},
\end{aligned}
$$

where $q^{2}=u_{i} u_{i}$. Thus when the flow in the region 1 is uniform the expressions for these quantities are known from the results of Sec. 6. These results moreover give the physical meaning of the invariants $B_{i j}$ and $d_{i}$. For example, $B_{31}$ and $B_{32}$ are the rates of change of half the square of the velocity magnitude along the lines of curvature as shown in (54).

Furthermore from (53) we get

$$
\begin{aligned}
p_{, i} p_{, i} & =\rho^{2} u_{n}^{2} B_{i 3} B_{k 3} D_{i i} D_{k i} \\
& =\rho^{2} u_{n}^{2}\left\{B_{13}^{2}+B_{23}^{2}+\left(-B_{13} \chi_{1}-B_{23} \chi_{2}+B_{33}\right)^{2}\right\} .
\end{aligned}
$$

Thus $p_{, i} \equiv 0$ only if $B_{13}=B_{23}=B_{33}=0$. But from (46), (47), and (12) we get

$$
B_{13}=\frac{4 \tau_{1} u_{1 n} k_{1}}{\gamma+1} ; \quad B_{23}=\frac{4 \tau_{2} u_{1 n} k_{2}}{\gamma+1} .
$$

Thus we find that Truesdell's [2] theorem in plane flows is carried over to spatial gas flows, viz., when a uniform flow of a fluid crosses a shock, the pressure gradient cannot vanish at any point on the rear side of the shock if the shock is curved and oblique.

8. Determination of the derivatives of entropy behind the shock. The value of the entropy $S$ as given by the relation (3) is

$$
S=J c_{v} \log \left(\frac{p}{\rho^{r}}\right) .
$$

Thus

$$
[S]=J c_{v} \log \left\{\frac{(1+\delta) p_{1}+\delta \rho_{1} u_{1 n}^{2}}{p_{1}(1+\delta)^{\gamma+1}}\right\}
$$


where we have used the shock relations (6) and (7).

In the case of uniform flow in front of the shock we derive, by differentiating both sides of (57), the result

$$
S_{. i} x_{\alpha}^{i}=\left\{J c_{v} \log \left(\frac{(1+\delta) p_{1}+\delta \rho_{1} u_{1 n}^{2}}{p_{1}(1+\delta)^{\gamma+1}}\right)\right\}_{;_{\alpha}}=E_{\alpha},
$$

where $E_{\alpha}$ can readily be evaluated with the help of the relations (15), (20) and (42). We also know that

$$
S_{. i} u_{i}=0 \text {, }
$$

which states that entropy is constant along the streamlines. This result and the relations (58) can be put in a compact form with the help of the matrix (26), as

$$
S_{. j} C_{i i}=f_{i} \text {, }
$$

where

$$
f_{1}=E_{1}, \quad f_{2}=E_{2}, \quad f_{3}=0 .
$$

Inverting the relation (59) with the help of the relation (27) we obtain

$$
S_{, j}=f_{i} D_{i j} \text {. }
$$

9. Curvature of the streamlines behind the shock. The curvature $K$ of a streamline in the flow under consideration is given by [8],

$$
K \mu_{i}=-\left(\frac{p_{. i}}{\rho q^{2}}+\frac{c^{2}}{q} u_{k, k} \lambda_{i}\right),
$$

where $\lambda_{i}$ and $\mu_{i}$ are the components of the unit tangent vector and principal normal vector to the streamlines. Hence

$$
K^{2}=\frac{1}{q^{4}}\left(\frac{p_{. i} p_{, i}}{\rho^{2}}-\frac{c^{4}}{q^{2}} u_{k, k}^{2}\right) .
$$

When we make use of the results (32) and (55) we get the value of $K^{2}$ as

$$
K^{2}=\frac{u_{n}^{2}}{q^{4}}\left\{B_{13}^{2}+B_{23}^{2}+\left(-B_{13} \chi_{1}-B_{23} \chi_{2}+B_{33}\right)^{2}-\frac{B_{33}^{2}}{1+\chi_{1}^{2}+\chi_{2}^{2}}\right\} .
$$

In this work we have confined ourselves to the evaluation of the first derivatives of the parameters of the flow behind the shock, while to determine the torsion of the streamlines we require as well the second derivatives [8] which have not yet been calculated.

10. The expression for the vorticity vector behind the shock. The components of vorticity behind the wave are given by

$$
w^{i}=e^{i j k} u_{k, i}=e^{i j k} B_{l m} D_{l k} D_{m i}=\left(-k_{1} v_{1} g_{11} \lambda_{2}^{i}+k_{2} v_{2} g_{22} \lambda_{1}^{i}\right) \frac{\delta^{2}}{1+\delta} .
$$

Now for $w^{i}$ to be zero it is necessary as well as sufficient that either $\delta^{2} /(1+\delta)$ or

$$
\left(-k_{1} v_{1} g_{11} \lambda_{2}^{i}+k_{2} v_{2} g_{22} \lambda_{2}^{i}\right)
$$

vanish. In the latter case coefficients of both $\lambda_{1}^{i}$ and $\lambda_{2}^{i}$ must vanish because $\lambda_{1}^{i}$ and $\lambda_{2}^{i}$ 
are perpendicular to each other. Thus in order that the flow be irrotational behind the shock surface at least one of the following eonditions must be satisfied

(ii) $\quad k_{1}$ and $k_{2}$ are zero, i.e., the shock surface is a plane;

(iii) $\quad v_{1}$ and $v_{2}$ are zero, i.e., the obliçuity is zero which again leads to a plane;

(iv) $k_{1}=0=v_{2}$;

(v) $\quad k_{2}=0=v_{1}$.

As (iv) and (v) lead to the same result, we will discuss, for the sake of definiteness, the case (v), viz., $k_{2}=0=v_{1}, k_{2}=0$ implies that the shock surface is a developable surface and as such its generators and their orthogonal trajectories form its two congruences of lines of curvature [5a]; while $v_{1}=0$ implies that the orthogonal trajectories to its generators are plane curves lying in the planes which are normal to the direction of the given uniform flow in front of the shock wave.

Now with the exception of cylinders and cones every developable surface is the tangent surface of some curve [5b] and the orthogonal trajectorics of the tangent surface of a curve are the involute of the curve [5c]. But the necessary and sufficient condition that the involutes of a twisted curve be plane curves is that the curve be a cylindrical helix [5d]. Moreover, the planes of the involutes of a cylindrical helix are normal to the generators of the cylinder on which the helix lies [5e]. Therefore, if we take the helix to lie on the cylinder whose generators are in the direction of the flow then the tangent surface of this helix, devclopable helicoid, satisfies the condition (v). As far as a cone and a cylinder are concerned it can be easily seen that the right circular cone with its axis parallel to the direction of the uniform flow and any cylinder with generators parallel to the direction of the uniform flow satisfy the conditions (v). We thus get the theorem: the only shock surfaces behind which the flow remains irrotational are, a plane, a right circular cone, a cylinder and a developable helicoid. Among these shocks the conical shocks have been extensively studied [9]. In the case of cylindrical shock surface whose generators are parallel to the direction of the uniform flow it is obvious that there is no discontinuity in the flow because flow is merely tangential to the surface.

Another result follows if we multiply both sides of the Eq. (62) by $\xi_{i}$ getting $w^{i} \xi_{i}=0$. Hence, when a uniform flow breaks across a shock, the component of vorticity along the normal to the shock wave vanishes at every point of the surface. It is interesting to note that this property is also shared by contact discontinuities, i.e., vortex sheets, which are singular surfaces across which the tangential component of the velocity changes abruptly while the normal component of velocity is continuous and the expression for vorticity components on the sheets is given by [10]

$$
w^{i}=e^{i j k} \xi_{j}\left[u_{k}\right],
$$

$\left[u_{k}\right]$ being the jump in velocity across the vortex-sheet. The component of vorticity normal to the three-dimensional pseudo-stationary and unsteady shock fronts has also been found to vanish $[11,12]$.

Acknowledgement. The author wishes to thank Professor 'T. Y. Thomas and Professor C. Truesdell for the interest they have shown in this work, and Professor D. Gilbarg for his helpful criticism. 
Note added in proof: The author has recently extended these results to unsteady shocks in fluids obeying an arbitrary equation of state [12].

\section{REFERENCES}

1. T. Y. Thomas, On curved shock waves, J. Math. Phys. 26, 62-68 (1947)

2. C. A. Truesdell, On curved shocks in steady plane flow of an ideal fluid, J. Aero. Sci. 19, 826-828 (1952)

3. R. Courant and K. O. Friedrichs, Supersonic flow and shock waves, Interscience Publishers, Inc., New York, 1948, Chap. I

4. I. Howarth, Ed., Modern developments in fluid dynamics, high speed flow, vol. I, Oxford, 1953, Chap. 2

5. L. P. Eisenhart, Introduction to differential geometry, Princeton Univ. Press, 1941: (a) Chap. 4, (b) p. 54, (c) p. 39 , (d) p. 38 , (e) p. 37

6. N. Coburn, Vector and tensor analysis, The Macmillan Co., New York, 1955, pp. 216-217

7. R. P. Kanwal, Shock and wave surfaces for three-dimensional rotational gas flows, $\mathrm{Ph} . \mathrm{D}$. thesis, Indiana University, 1957

8. R. P. Kanwal, Variation of flow quantities along streamlines, their principal normals and binormals in three-dimensional gas flows, to appear in the September 1957 issue of the J. Math. Mech.

9. G. I. Taylor and J. W. Maccoll, The air pressure on a cone moving at high speeds, Proc. Roy. Soc. A139, 278-311 (1933)

10. L. M. Milne-Thompson, Theoretical aerodynamics, Macmillan and Co., London, 1948, p. 171

11. R. P. Kanwal, Propagation of curved shock waves in pseudo-stationary three-dimensional gas flows, Illinois Journal of Mathematics, 2, 129-136 (1958)

12. R. P. Kanwal, Determination of vorticity and gradients of flow parameters behind a three-dimensional unsteady curved shock wave, Arch. Ratl. Mech. Anal. (in press) 